\title{
Performance measures for in-hospital care of acute ischemic stroke in public hospitals in Chile
}

\author{
Lorena Hoffmeister ${ }^{1 *}$, Pablo M Lavados ${ }^{2,3}$, Merce Comas ${ }^{4}$, Carolina Vidal ${ }^{1}$, Rodrigo Cabello ${ }^{1}$ and Xavier Castells ${ }^{4}$
}

\begin{abstract}
Background: The aim of this study were to describe acute care of ischemic stroke patients and adherence to performance measures, as well as the outcomes of these events, in a sample of patients treated in public hospitals in Chile.

Methods: We retrospectively reviewed the medical charts of patients with ischemic stroke from a sample of seven public hospitals in the Metropolitan Region of Santiago. We analyzed adherence to the following evidence-based measures: clinical evaluation at admission, use of intravenous thrombolysis, dysphagia screening and prescription of antithrombotic therapy at discharge. As outcome measures we analyzed post-stroke pneumonia and 30-day casefatality. We used a logistic regression model by each outcome with generalized estimating equations, which accounted for clustering of patients within hospitals and included sex, age (years), clinical status at admission (reduced level of consciousness, speech disturbance, aphasia and hemiplegia), comorbidities, dysphagia screening and neurological evaluation at admission as measures of acute stroke care.
\end{abstract}

Results: We reviewed the charts of 677 patients, of which 52.3\% were men. The mean age was 69.8 years in women and 66.3 years in men. Diagnosis of stroke was confirmed by a computed tomography scan within 4.5 hours of symptom onset in only $9.6 \%$ of the patients. Intravenous thrombolysis was administered in $1.7 \%$. Dysphagia screening was performed in $12.1 \%$ (95\% Cl 9.7-15.0) and antithrombotic therapy was prescribed in 68.9\% (95\% Cl 64.6-72.9). Pneumonia was diagnosed in $23.6 \%$ (95\% Cl 20.4-27.2). Thirty-day fatality was $8.7 \%$ (95\% Cl 6.7-11.3). The variables independently associated with 30-day case fatality were age (OR 1.08, 95\% 1.06-1.10), pneumonia (OR 7.7, 95\% 95\% Cl 4.0-14.7), aphasia (OR 2.4, 95\% Cl 1.1-5.6), reduced level of consciousness (OR 2.4, $95 \% \mathrm{Cl} 1.3-4.4)$, and speech disturbance (OR 1.4, 95\% Cl 1.0-1.9). No association was found between 30-day case fatality and dysphagia screening or neurological evaluation at admission. The factors associated with post-stroke pneumonia were female sex (OR 1.6, 95\% Cl 1.0-2.3), age (OR 1.04 95\% Cl 1.03-1.05), diagnosis of diabetes (OR 1.8, 95\% Cl 1.4-2.4), aphasia (OR 2.0, 95\% Cl 1.5-2.7), hemiplegia (OR 1.6, 95\% Cl 1.1-2.4), and reduced level of consciousness on admission (OR 3.4, 95\% Cl 2.1-5.5). No association was found between pneumonia and dysphagia screening or neurological evaluation at admission.

Conclusions: Adherence to evidence-based performance measures was low. Administration of intravenous thrombolysis was particularly low and diagnostic confirmation of ischemic stroke was delayed. The occurrence of post-stroke pneumonia was frequent and should be reduced. To improve acute stroke care in Chile, organizational change in the health service is urgently needed.

Keywords: Acute ischemic stroke, Stroke care, Thrombolysis

\footnotetext{
* Correspondence: lorena.hoffmeister@umayor.cl

${ }^{1}$ School of Public Health, Facultad de Medicina, Universidad Mayor, Santiago, Chile

Full list of author information is available at the end of the article
}

\section{Biomed Central}

(c) 2013 Hoffmeister et al.; licensee BioMed Central Ltd. This is an Open Access article distributed under the terms of the Creative Commons Attribution License (http://creativecommons.org/licenses/by/2.0), which permits unrestricted use distribution, and reproduction in any medium, provided the original work is properly cited. 


\section{Background}

The global burden of stroke is greater in middle and low-income than in high-income countries [1,2]. In Chile, the annual adjusted incidence of stroke is 97.4 per 100,000 inhabitants [3], and these events cause $9 \%$ of all deaths. In the population aged 45-84 years, $65 \%$ of strokes are ischemic, corresponding to an incidence rate for a first event of 59.6 per 100,000 inhabitants, with a mean age of 68.6 years in women and 64.7 in men [4]. Only a few interventions have been demonstrated to improve prognosis after an acute ischemic stroke. Such interventions include admission to an organized Stroke Unit, which reduces death and disability and cost in all types of stroke irrespective of age or sex [5], and enhancing adherence to important clinical practice guideline recommendations [6]. The chance of a good outcome at 6 months is increased by aspirin administration within 48 hours of symptom onset, while, in large hemispheric infarctions, 3-month mortality is decreased by hemicraniectomy within 48 hours of symptom onset to prevent brain herniation [7].

The only specific drug treatment with proven costeffectiveness in reducing the disability caused by ischemic stroke is intravenous thrombolysis with recombinant tissue plasminogen activator (rtPA) [8,9]. The effectiveness of this procedure decreases according to the interval between stroke onset and the start of treatment, which is not recommended after 270 minutes [10,11]. National [12] and international clinical guidelines recommend the use of thrombolysis in the acute management of ischemic stroke $[9,13,14]$. Although these recommendations were made several years ago, studies in high-income countries have reported low use of intravenous thrombolysis, even among patients admitted to hospital within the therapeutic window [15]. In Australia, intravenous thrombolysis is used in $7 \%$ of all patients with ischemic stroke, mainly in hospitals with stroke units [16]. A study performed in the USA found that intravenous thrombolysis was used in $2.4 \%$ of admissions for ischemic stroke [17]. Isolated publications in low-middle income countries have reported that the use of this treatment is even lower [18].

The systematic use of clinical guidelines depends largely on health service organization. In the USA, the use of intravenous thrombolysis and other evidencebased performance measures has significantly improved in hospitals adhering to the Get with the GuidelinesStroke program $[19,20]$. In Spain, a Stroke Program audit of the quality of in-hospital stroke care reported significant improvements in preventive measures for concomitant conditions or potential complications, as well as in the early evaluation of the need for care [21]. After stroke services were redesigned in Australia, brain imaging within 24 hours after stroke and prescription of anti-thrombotic therapy at discharge significantly increased [6].

In Chile, the system of Explicit Health Guarantees (EHG) for ischemic stroke was implemented in 2005, which guaranteed access to confirmation of diagnosis and acute treatment of strokes to $91.0 \%$ of the Chilean population, including clinical practice guidelines with evidence-based recommendations [12]. The development of organized Stroke Units is only now starting to happen in Chile and was not included as part of the 2005 EHG for ischemic stroke.

The aim of this study was to assess acute care of ischemic stroke and adherence to performance measures, as well as to identify the outcome of these events, in a sample of patients treated in public hospitals in Chile from 2007 to 2009.

\section{Methods}

We retrospectively reviewed the medical charts of a sample of patients aged 15 years and above, hospitalized with a diagnosis of ischemic stroke in 7 out of 22 public hospitals of the Metropolitan Region of Santiago, Chile. Overall, the selected hospitals provide health care to an adult population of 5,375,908 persons, representing $40.4 \%$ of the Chilean population. In 2009, these hospitals received $85.3 \%$ of all admissions for stroke in the Metropolitan Region of Santiago and 55.3\% of those in the country as a whole, admitted 150 to 878 patients per year. These hospitals are referral centers for stroke care in each of the six territorially organized health services of the public health care network in the Metropolitan Region of Santiago. All study hospitals had adult emergency services and access to on-site computed tomography (CT) 24 hours per day and 365 days per year. We excluded pediatric hospitals $(n=5)$, subspecialty institutes $(n=7)$, hospitals without emergency departments $(n=3)$, and community hospitals without CT scanners $(n=8)$. The sampling frame was the Management System of the Explicit Health Guarantees, which is mandated to routinely register all patients with ischemic stroke admitted to hospitals in the public sector. All patients with a main admission diagnosis of ischemic stroke (ICD-10 I63) between August 2007 and August 2009 were included. The patients were identified through their national identification number, sex, age at event, and the admitting hospital. The study protocol was approved by the Ethics Committee of the Faculty of Medicine of the Universidad Mayor, Santiago, Chile.

\section{Data collection}

A structured case report form was designed and tested in three hospitals in the Metropolitan Region of Santiago. Data were extracted by a team of researchers external to the selected hospitals, composed of clinical nurses with experience in adult emergency departments. All nurses 
were trained in the protocol and calibration was performed. If the measures were not documented, they were considered not to have been performed.

To check the quality of data collection, an external audit was carried out by blinded and independent researchers. This audit consisted of double data collection of approximately $10 \%$ of the patients admitted to each hospital and selected randomly from the patient lists. To determine consistency among raters, interrater reliability was analyzed using the Kappa statistic.

\section{Study variables}

The variables were selected based on a review of the literature on the acute management of ischemic stroke, evidence-based recommendations in protocols and clinical practice guidelines, expert evaluation (neurologists, clinical epidemiologists and emergency department nurses), and the availability of data in the medical chart. We chose the performance measures that we believed to be most indicative of the outcomes of case fatality and pneumonia. Adherence to the following performance measures was included: a) completeness of the data and time from symptom onset to CT confirmation of the ischemic stroke diagnosis by neurologist, b) the time interval from symptom onset to CT scan and diagnostic confirmation of ischemic stroke by neurologist, c) the percentage of patients who underwent dysphagia screening through a simple, valid bedside test before receiving any food, fluids or medication and within 48 hours of hospital admission (excluding comatose patients), d) the proportion of patients with neurological evaluation on admission through the Glasgow Coma Scale [22], 3) the proportion of ischemic stroke patients with prescribed antithrombotic therapy at hospital discharge, and f) the percentage of patients administered intravenous thrombolysis. We also included the occurrence of post-stroke pneumonia diagnosed after hospital admission, and 30-day case fatality. Pneumonia was defined as pneumonia occurring during hospitalization, documented by a physician, and requiring antibiotic treatment.

Because patients can die after hospital discharge, survival was determined through the link between the variables identifying ischemic stroke patients (complete name and unique identity number) and the official mortality register. This register is exhaustive and the cause of death was medically certified in $99.3 \%$ of deaths in 2007, while $2.9 \%$ of deaths were classified as poorly defined [23]. The death coding process is carried out according to standardized and centralized criteria in the Statistics and Health Information Department of the Ministry of Health and the main cause of death is coded according to the ICD-10 classification.

\section{Statistical analysis}

The sample size was calculated to provide an estimated $15.0 \%$ prevalence of in-hospital pneumonia, 2.5\% precision, a 95\% confidence interval, and $12 \%$ predicted losses. A population of 10,467 ischemic stroke patients in the Metropolitan Region of Santiago was used to estimate the sample. The overall sample consisted of 821 patients. Due to differences in the volume of patients admitted to the study hospitals, stratified random sampling by hospital (stratum) was used to select ischemic stroke patients from the list of patients. In each hospital, an equal number of patients $(n=117)$ were randomly sampled, except in one hospital where 119 patients were selected. Thus, the probability of including a patient in the sample depended on the hospital's size. According to the sample design and to obtain representative results, sampling weights were calculated as the inverse probability of being included in the study. Descriptive results were weighted.

Performance measures were calculated by taking the number of patients receiving a particular intervention as the numerator, and the total number of patients eligible for this intervention as the denominator. Proportions and $95 \%$ confidence intervals were calculated for the population characteristics and for the study variables. Proportions were compared among hospitals by using the Kruskal-Wallis test.

To estimate the adjusted odds ratios of 30-day fatality and in-hospital pneumonia according to adherence to selected performance measures, dysphagia screening and neurological evaluation, we used multivariable logistic regression with generalized estimating equations (GEE), which accounted for the correlations among patients within hospitals. The model for 30-days fatality was adjusted by the patients' demographic characteristics (sex and age at event), comorbidities (diabetes and hypertension), in-hospital pneumonia (because it is an important predictor of post-stroke fatality) [24] and clinical status at admission (aphasia, hemiplegia, reduced level of consciousness, and speech disturbance). These variables showed the degree of neurological impairment assessed within the first few hours after arrival at the hospital and were included in the regression model as proxies of stroke severity [25]. The model for post-stroke pneumonia was adjusted using the same patient's characteristics, and excluding pneumonia. A p-value of $<0.05$ was considered statistically significant and odds ratios with their 95\% CI were calculated.

Potential selection biases were assessed by comparing the distributions of sex, mean age, and the proportion of 30-day fatality among participants and non-participants in the study. The Chi-squared test was used to compare differences in sex and 30-day fatality, and Student's $t$-test was used to compare age. 
All statistical analyses were performed using the Statistical Package for the Social Sciences (SPSS) program, version 20.0.

\section{Results}

Of 821 patients selected, 677 were included in the study. We excluded 124 patients because their medical charts could not be found after five searches on different dates or because the charts contained insufficient information for diagnosis of an ischemic stroke (Figure 1). Twenty patients without confirmed ischemic stroke were also excluded. The overall interrater agreement was 0.75 , and more than $85 \%$ of the variables achieved kappa values above 0.6 (using the Landis \& Koch classification) [26]. The number of excluded patients was significantly greater in hospital 2 than in the remaining hospitals. Overall, there were no significant differences between participants and non-participants in sex distribution (p-value: 0.84 ), mean age (p-value: 0.60 ), or 30 -day fatality (p-value: 0.27 ).

Men accounted for $52.3 \%$ of the sample (Table 1). The mean age was 69.8 years (95\% CI 68.0- 71.6) in women and 66.3 years ( $95 \%$ CI 64.9- 67.6) in men. A history of hypertension was found in $77.0 \%$ and a diagnosis of diabetes mellitus in $28.5 \%$. There were no significant differences among hospitals in patient characteristics (Table 2).

The percentage of patients with complete information on the date and time of symptom onset was $47.0 \%$ (95\% CI 42.9-51.1). Significant differences were found among hospitals ( $\mathrm{p}<0.05$ ), with a range of between $36.7 \%$ and
Table 1 Characteristics of the study population

\begin{tabular}{lll}
\hline & $\mathbf{n}$ & Percentage $\mathbf{( 9 5 \% ~ C l )}$ \\
\hline Sex & 359 & $52.3(48.2-56.4)$ \\
\hline Male & 318 & $47.7(43.6-51.8)$ \\
\hline Age & & \\
\hline$<=50$ years & 82 & $12.6(10.1-15.7)$ \\
\hline 51 - 60 years & 91 & $13.9(11.3-17.0)$ \\
\hline 61 - 70 years & 180 & $26.4(23.0-30.2)$ \\
\hline 71- 80 years & 206 & $30.2(26.5-34.1)$ \\
\hline More than 80 years & 114 & $16.9(14.0-20.2)$ \\
\hline Comorbidities & & \\
\hline Diagnosis of diabetes & 197 & $28.5(25.0-32.3)$ \\
\hline History of hypertension & 533 & $77.0(73.3-80.4)$ \\
\hline Hospitals & & \\
\hline Hospital 1 & 99 & 25.5 \\
\hline Hospital 2 & 64 & 8.6 \\
\hline Hospital 3 & 110 & 13.0 \\
\hline Hospital 4 & 98 & 3.8 \\
\hline Hospital 5 & 98 & 12.6 \\
\hline Hospital 6 & 105 & 18.9 \\
\hline Hospital 7 & 103 & 17.7 \\
\hline
\end{tabular}

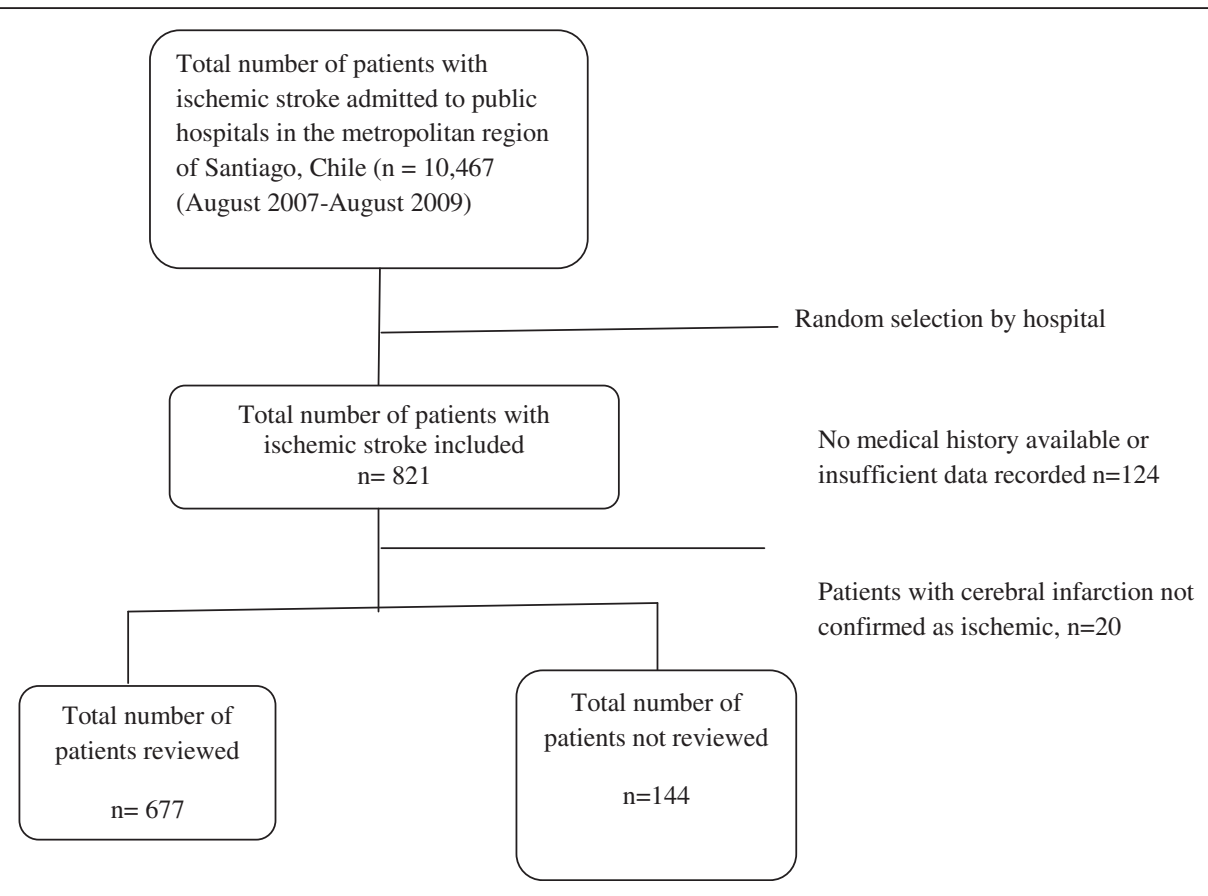

Figure 1 Flow chart of patient inclusion in the study. 
Table 2 Characteristics of the study population by hospital

\begin{tabular}{|c|c|c|c|c|c|c|c|c|c|c|c|c|c|c|c|c|c|c|c|c|c|c|c|}
\hline & & \multicolumn{3}{|c|}{ Hospital 1} & \multicolumn{3}{|c|}{ Hospital 2} & \multicolumn{3}{|c|}{ Hospital 3} & \multicolumn{3}{|c|}{ Hospital 4} & \multicolumn{3}{|c|}{ Hospital 5} & \multicolumn{3}{|c|}{ Hospital 6} & \multicolumn{3}{|c|}{ Hospital 7} & \multirow{3}{*}{$\begin{array}{c}\text { Kruskall- } \\
\text { Wallis } \\
\text { p-value }\end{array}$} \\
\hline & & \multirow[t]{2}{*}{$\%$} & \multicolumn{2}{|c|}{$\mathrm{Cl}(95 \%)$} & \multirow[t]{2}{*}{$\%$} & \multicolumn{2}{|c|}{$\mathrm{Cl}(95 \%)$} & \multirow[t]{2}{*}{$\%$} & \multicolumn{2}{|c|}{$\mathrm{Cl}(95 \%)$} & \multirow[t]{2}{*}{$\%$} & \multicolumn{2}{|c|}{$\mathrm{Cl}(95 \%)$} & \multirow[t]{2}{*}{$\%$} & \multicolumn{2}{|c|}{$\mathrm{Cl}(95 \%)$} & \multirow[t]{2}{*}{$\%$} & \multicolumn{2}{|c|}{$\mathrm{Cl}(95 \%)$} & \multirow[t]{2}{*}{$\%$} & \multicolumn{2}{|c|}{$\mathrm{Cl}(95 \%)$} & \\
\hline & & & Lower & Upper & & Lower & Upper & & Lower & Upper & & Lower & Upper & & Lower & Upper & & Lower & Upper & & Lower & Upper & \\
\hline \multirow[t]{3}{*}{ Sex } & Men & 47.5 & 37.7 & 57.5 & 57.8 & 45.1 & 69.5 & 47.3 & 38.1 & 56.8 & 55.1 & 45.0 & 64.8 & 58.2 & 48.0 & 67.7 & 57.1 & 47.4 & 66.4 & 50.5 & 40.8 & 60.2 & 0.487 \\
\hline & Women & 52.5 & 42.5 & 62.3 & 42.2 & 30.5 & 54.9 & 52.7 & 43.2 & 61.9 & 44.9 & 35.2 & 55.0 & 41.8 & 32.3 & 52.0 & 42.9 & 33.6 & 52.6 & 49.5 & 39.8 & 59.2 & \\
\hline & Total & 100 & & & 100 & & & 100 & & & 100 & & & 100 & & & 100 & & & 100 & & & \\
\hline \multirow[t]{6}{*}{ Age (years) } & $<=50$ & 15.8 & 9.7 & 24.7 & 18.8 & 10.8 & 30.5 & 7.3 & 3.6 & 14.0 & 13.3 & 7.8 & 21.7 & 8.2 & 4.1 & 15.7 & 11.4 & 6.5 & 19.2 & 13.6 & 8.1 & 21.8 & 0.219 \\
\hline & $51-60$ & 12.6 & 7.2 & 21.1 & 12.5 & 6.3 & 23.4 & 10.9 & 6.2 & 18.4 & 10.2 & 5.5 & 18.1 & 17.3 & 11.0 & 26.3 & 13.3 & 8.0 & 21.4 & 17.5 & 11.2 & 26.2 & \\
\hline & $61-70$ & 25.3 & 17.4 & 35.1 & 23.4 & 14.5 & 35.7 & 29.2 & 21.3 & 38.5 & 29.6 & 21.3 & 39.5 & 22.4 & 15.2 & 31.9 & 31.4 & 23.2 & 41.1 & 24.3 & 16.9 & 33.6 & \\
\hline & 71- 80 & 28.4 & 20.1 & 38.5 & 32.8 & 22.2 & 45.5 & 28.2 & 20.4 & 37.4 & 32.7 & 24.0 & 42.7 & 31.6 & 23.1 & 41.6 & 26.7 & 19.0 & 36.1 & 35.0 & 26.2 & 44.8 & \\
\hline & 80 and older & 17.9 & 11.3 & 27.1 & 12.5 & 6.3 & 23.4 & 24.5 & 17.3 & 33.6 & 14.3 & 8.6 & 22.9 & 20.4 & 13.5 & 29.7 & 17.1 & 11.0 & 25.7 & 9.7 & 5.2 & 17.3 & \\
\hline & Total & 100 & & & 100 & & & 100 & & & 100 & & & 100 & & & 100 & & & 100 & & & \\
\hline \multirow[t]{3}{*}{ Diabetes } & $\begin{array}{l}\text { Without } \\
\text { diagnostic }\end{array}$ & 72.7 & 63.0 & 80.7 & 68.8 & 56.1 & 79.1 & 69.1 & 59.8 & 77.1 & 69.4 & 59.4 & 77.8 & 70.4 & 60.5 & 78.7 & 78.1 & 69.0 & 85.1 & 67.0 & 57.2 & 75.5 & 0.678 \\
\hline & $\begin{array}{l}\text { With } \\
\text { diagnostic }\end{array}$ & 27.3 & 19.3 & 37.0 & 31.3 & 20.9 & 43.9 & 30.9 & 22.9 & 40.2 & 30.6 & 22.2 & 40.6 & 29.6 & 21.3 & 39.5 & 21.9 & 14.9 & 31.0 & 33.0 & 24.5 & 42.8 & \\
\hline & Total & 100 & & & 100 & & & 100 & & & 100 & & & 100 & & & 100 & & & 100 & & & \\
\hline \multirow[t]{3}{*}{ Hypertension } & $\begin{array}{l}\text { Without } \\
\text { diagnostic }\end{array}$ & 31.3 & 22.8 & 41.3 & 20.3 & 12.0 & 32.3 & 19.1 & 12.7 & 27.6 & 16.3 & 10.2 & 25.2 & 20.4 & 13.5 & 29.7 & 24.8 & 17.3 & 34.1 & 16.5 & 10.4 & 25.1 & 0.128 \\
\hline & $\begin{array}{l}\text { With } \\
\text { diagnostic }\end{array}$ & 68.7 & 58.7 & 77.2 & 79.7 & 67.7 & 88.0 & 80.9 & 72.4 & 87.3 & 83.7 & 74.8 & 89.8 & 79.6 & 70.3 & 86.5 & 75.2 & 65.9 & 82.7 & 83.5 & 74.9 & 89.6 & \\
\hline & Total & 100 & & & 100 & & & 100 & & & 100 & & & 100 & & & 100 & & & 100 & & & \\
\hline
\end{tabular}


$58.1 \%$. In $58.2 \%$ of patients (95\% CI 54.2-62.2), the date and time of CT scans and diagnostic confirmation by a neurologist was recorded. Recording of the date and time of CT scans was significantly lower in two hospitals than in the remaining hospitals $(\mathrm{p}<0.05)$.

A CT scan was performed within 24 hours of symptom onset in only $45.2 \%$ of patients, while the interval between symptom onset and CT scan could not be determined in $21.1 \%$ of the medical charts (95\% CI 17.8 24.9). An interval of up to 270 minutes between symptom onset and CT-based confirmation of the diagnosis occurred in $9.6 \%$ of the patients (95\% CI 7.4-12.2). This percentage varied among hospitals, ranging from $3.6 \%$ (95\% CI 1.4-9.4) to $14.3 \%$ (95\% CI 8.6-22.7) (Figure 2).

Intravenous thrombolysis was used in 15 patients in only two hospitals (Table 3), representing 1.7\% (95\% CI 1.0-3.0). Neurological evaluation on admission using a standard clinical scale was performed in 52.4\% (95\% CI 48.3-56.5), with significant differences among hospitals $(\mathrm{p}=0.03)$. Dysphagia screening was carried out in $12.1 \%$ (95\% CI 9.7-15.0) of patients with indication for this procedure, with significant differences among hospitals, ranging from $4.2 \%$ to $25.4 \%$ ( $\mathrm{p}<0.0001$ ). Antithrombotic therapy was prescribed at discharge in 68.9\% (95\% CI 64.6-72.9), with significant differences among hospitals ( $\mathrm{p}<0.0001$ ). In-hospital pneumonia occurred in $23.6 \%$ (95\% CI 20.4-27.2), but this percentage was much higher in hospital 3: 41.9\% (95\% CI 32.9-51.4) compared with the remaining hospitals $(\mathrm{p}<0.0001)$. Case-fatality at $30-$ days was $8.7 \%$ (95\% CI 6.7-11.3) with significant differences among centers $(\mathrm{p}<0.0001)$, hospital 5 showing the highest mortality (Figure 3 ).
The GEE for 30-day case-fatality (Table 4), which included the effect of clustering patients in hospital, showed that demographic and clinical variables independently associated with an increased probability of death were age (OR 1.08, 95\% CI 1.06-1.10), post-stroke pneumonia (OR 7.65, 95\% CI 3.98-14.72), and reduced level of consciousness (OR- 2.41, 95\% CI 1.32-4.41) at hospital admission. No association was found between the performance of neurological evaluation (OR 2.02, 95\% CI 0.77-5.30) and dysphagia screening (OR 0.52, 95\% CI 0.26-1.04) and the likelihood of 30-day fatality.

For post-stroke pneumonia (Table 4), the demographic and clinical variables independently associated with an increased risk of pneumonia were female sex (OR 1.55, 95\% CI 1.03-2.33), age (OR 1.04, 95\% CI 1.03-1.05), diabetes (OR 1.79, 95\% CI 1.35-2.38), aphasia (OR 1.97, 95\% CI 1.45-2.69), hemiplegia (OR 1.60, 95\% CI 1.082.38 ), and reduced level of consciousness (OR 3.37, CI 95\% 2.07-5.48). No association was found between dysphagia screening and neurological evaluation and poststroke pneumonia.

\section{Discussion}

In this study, we found that adherence to evidencebased performance measures in the acute care of ischemic stroke in a sample of public hospitals in the Metropolitan Region of Santiago was very low. Several programs to improve the quality and delivery of care have prospectively investigated performance measures before and after intervention programs around the world and have described similar results before organizational interventions in acute stroke care [20,21,27-30].

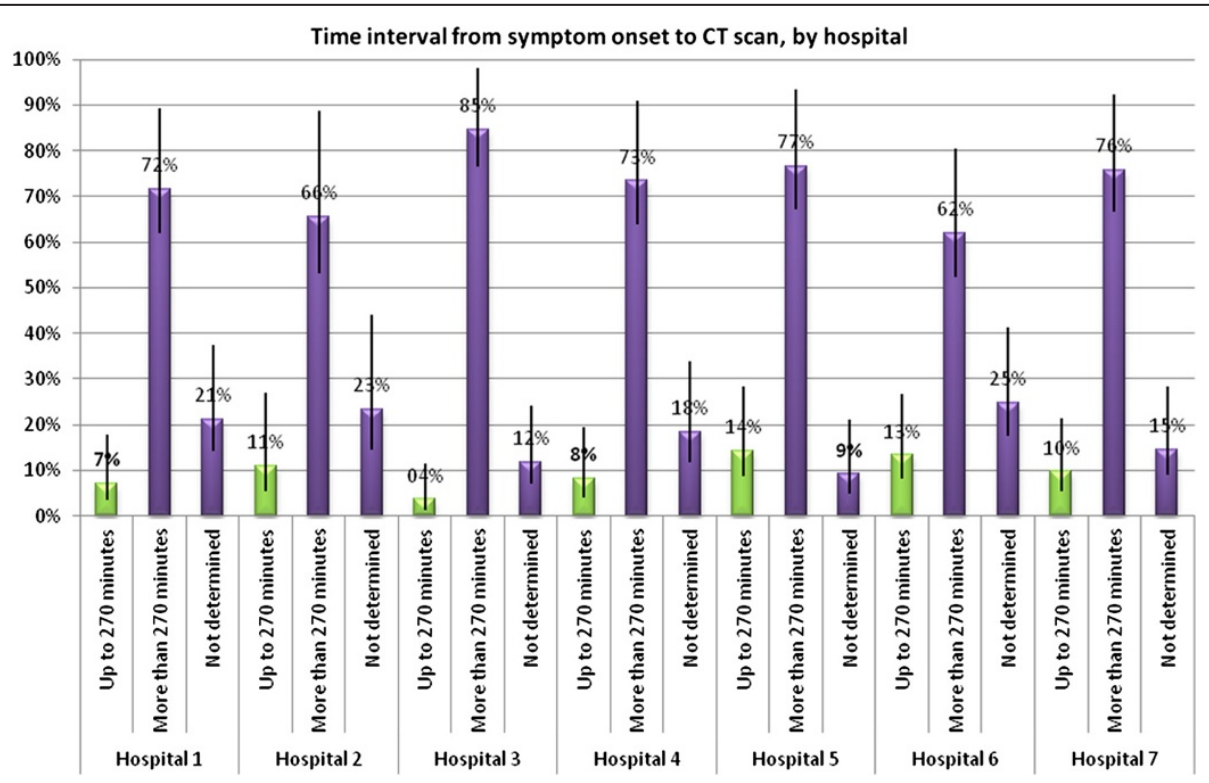

Figure 2 Time interval from symptom onset to CT scan by hospital. 
Table 3 Performance measures, percentage of patients with pneumonia and 30-days case fatality

\begin{tabular}{lll}
\hline Measure & Number of interventions performed/number indicated & \% (95\% Cl) \\
\hline Percentage of patients with neurological evaluation on admission & $389 / 677$ & $52.4(48.3-56.5)$ \\
\hline Dysphagia screening & $84 / 609^{*}$ & $12.1(9.7-15.0)$ \\
\hline Prescription of antithrombotics at discharge & $457 / 612^{\dagger}$ & $68.9(64.6-72.9)$ \\
\hline Intravenous thrombolysis & $15 / 677$ & $1.70(1.0-3.0)$ \\
\hline In-hospital pneumonia & $167 / 677$ & $23.6(20.4-27.2)$ \\
\hline 30-day case fatality & $59 / 677$ & $8.7(6.7-11.3)$ \\
\hline
\end{tabular}

"68 patients who were unconscious at hospital admission were excluded.

${ }^{+}$Patients who died during their hospital stay were excluded.

In our study, a CT scan was performed within 24 hours of symptom onset in less than $50 \%$ of patients and within 4.5 hours in less than $10 \%$, limiting the possibility of acute care, especially intravenous thrombolysis or hemicraniectomy. The literature reveals considerable variability in arrival times to hospital. In the Paul Coverdell Registry, the median time from hospital arrival to brain imaging among all patients was 1 hour, 12 minutes (mean: 2 hours, 36 minutes) [27], while in Korea the median time interval from symptom onset to hospital arrival was 7 hours, 54 minutes [31]. Consistent with the finding of delays from symptom onset to CT confirmation of diagnosis, intravenous thrombolysis was administered in only $1.7 \%$ of the patients, a lower percentage than that in high-income countries $[16,19]$, and similar to that reported in Argentina and China, where $1.05 \%$ and $2.4 \%$ of patients with acute ischemic stroke were treated with intravenous thrombolysis [30,32]. This low use was to be expected, since systematic organizational changes have not been implemented in hospitals and thrombolysis is usually administered in hospitals with stroke units [16].

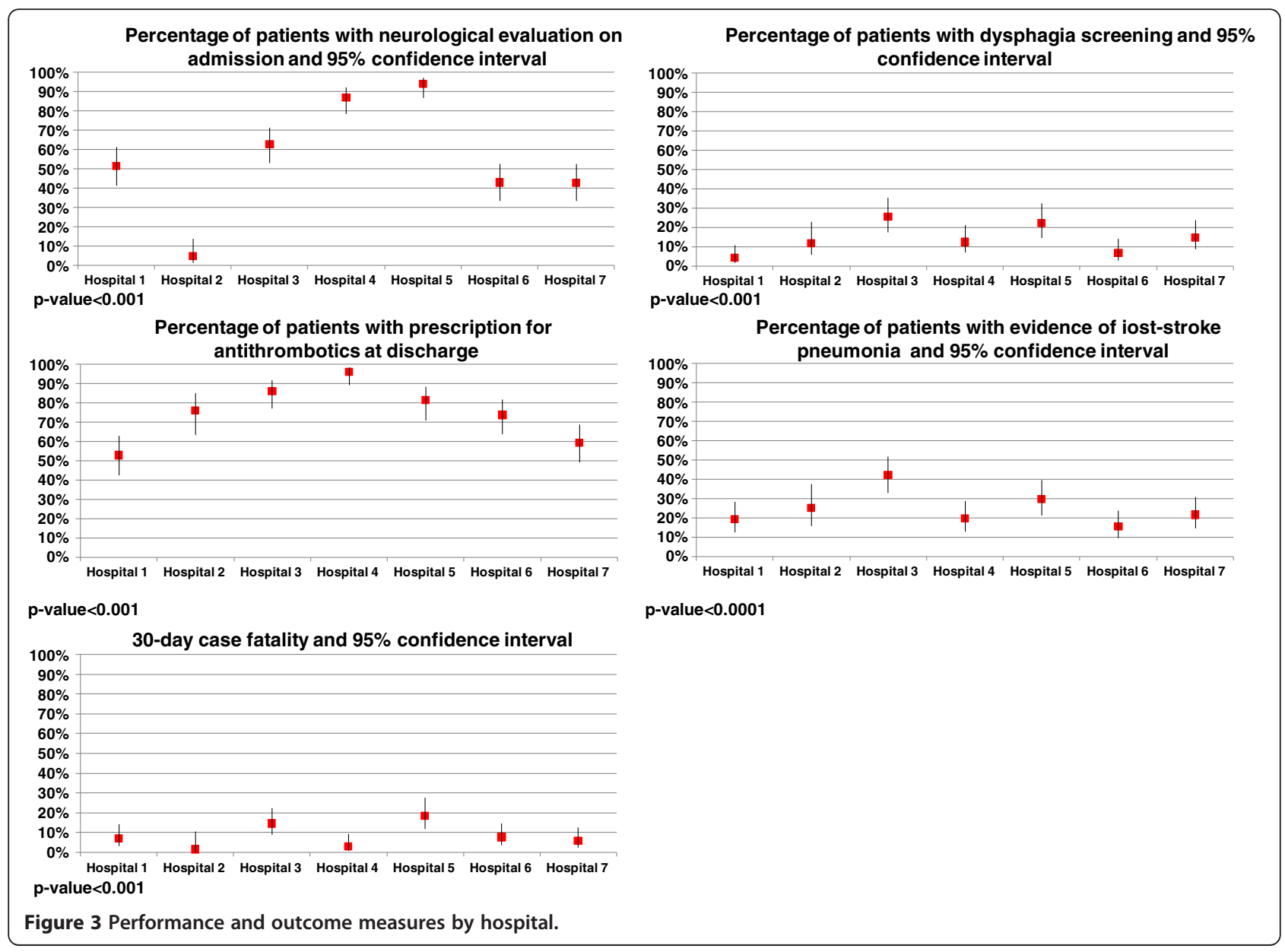


Table 4 Factors associated with 30-day case-fatality and in-hospital pneumonia, adjusted odds ratios from generalized estimating equations

\begin{tabular}{|c|c|c|c|c|}
\hline & \multicolumn{2}{|c|}{ 30-day case-fatality } & \multicolumn{2}{|c|}{ In-hospital pneumonia } \\
\hline & Adjusted OR (95\% CI) & p-value & Adjusted OR (95\% Cl) & p-value \\
\hline Sex: female & $1.48(0.82-2.27)$ & 0.28 & $1.55(1.03-2.33)$ & 0.03 \\
\hline Age (years) & $1.08(1.06-1.10)$ & 0.00 & $1.04(1.03-1.05)$ & 0.00 \\
\hline Diagnosis of diabetes & $0.53(0.24-1.15)$ & 0.11 & $1.79(1.35-2.38)$ & 0.00 \\
\hline Diagnosis of hypertension & $1.90(0.49-7.30)$ & 0.35 & $0.82(0.54-1.24)$ & 0.33 \\
\hline Aphasia on admission & $2.42(1.05-5.61)$ & 0.04 & $1.97(1.45-2.69)$ & 0.00 \\
\hline Hemiplegia on admission & $0.57(0.23-1.39)$ & 0.21 & $1.60(1.08-2.38)$ & 0.02 \\
\hline Reduced level of consciousness on admission & $2.41(1.32-4.41)$ & 0.00 & $3.37(2.07-5.48)$ & 0.00 \\
\hline Speech disturbance on admission & $1.39(1.02-1.90)$ & 0.04 & $1.24(0.88-1.74)$ & 0.22 \\
\hline Post-stroke pneumonia & 7.65 (314.72) & 0.00 & - & - \\
\hline Neurological evaluation & $2.02(0.77-5.30)$ & 0.15 & $1.07(0.79 ; 1.44)$ & 0.66 \\
\hline Dysphagia screening & $0.52(0.26-1.04)$ & 0.07 & $1.58(0.60 ; 4.15)$ & 0.36 \\
\hline
\end{tabular}

Models adjusted by all factors in this table and clustering of patients by individual hospital.

We detected substantial deficiencies in the thoroughness and accuracy of registration of the times of symptom onset and CT confirmation of diagnosis. Monitoring the time from symptom onset to the distinct interventions is essential to improve patient care and adherence to evidence-based performance measures in the acute management of stroke and to reduce pre-admission and post-admission barriers to treatment [15]. Of 56,969 patients in the Paul Coverdell Registry, the time from symptom onset to hospital arrival was unknown or not recorded in 57.8\% [27].

This study shows that clinical neurological evaluation measures such as the National Institute of Health Stroke Scale (NIHSS) have not been incorporated in routine clinical practice in the emergency departments of the hospitals in the sample. The NIHSS has established validity and reliability for use in the clinical evaluation of stroke patients and is strongly predictive of early functional recovery and long-term outcome. This scale can be administered by physicians, research workers, and nurses alike and has proven intra- and inter-rater reliability [33]. In addition to the low use of the NIHSS, we also found that early evaluation of neurological deficit was not systematically performed in all the hospitals studied.

Dysphagia screening was particularly low in our study and could strongly influence the incidence of pneumonia. In the Canadian Stroke Network Registry, dysphagia screening was performed in $56 \%$ of patients [28]. A study using the Paul Coverdell Registry showed that unscreened patients were at a higher risk of pneumonia than screened patients [34].

In the present study, more than one-fifth of the patients developed in-hospital pneumonia. Post-stroke pneumonia is a potentially preventable complication that is associated with poor outcome [35]. In the multivariable analysis, the factors associated with pneumonia were age, female sex, a reduced level of consciousness on admission, aphasia, hemiplegia and a diagnosis of diabetes. Neither dysphagia screening nor clinical evaluation care contributed significantly to post-stroke pneumonia. Dysphagia screening was carried out in a very small proportion of patients and could not fully be evaluated in the multivariable model. Clinical evaluation on admission, as defined in current clinical practice, was insufficiently specific to identify other variables with proven prognostic value in the risk of post-stroke pneumonia, such as pre-existing dependency, non-lacunar vs lacunar stroke, chronic obstructive pulmonary disease and other factors [36]. Finlayson reported that health care determinants, such as stroke unit admission did not predict the occurrence of pneumonia but were, however, associated with decreased mortality from this infection, indicating the need to intensify acute stroke care [36]. Like Aslayen et al. [37], we found an increased risk of pneumonia in women and patients with diabetes, which could be explained by the higher risk of infections in diabetic patients.

Among secondary prevention measures, compliance with recommendations on the prescription of antithrombotic agents at discharge was lower than expected and these drugs were not prescribed in a third of the eligible patients; variations among hospitals were also found. This finding is particularly worrisome as prescription of these drugs at discharge is one of the most widely used and cheapest secondary preventive measures. In most registries, compliance with this performance measure was usually above $90 \%$ in both high- and low-medium income countries $[20,21,30]$. Likewise, no record was found in the medical charts of disability evaluation at discharge or the need for community- 
based rehabilitation. Although not usually considered performance measures of acute stroke care, both measures are associated with improved outcomes [38].

The case fatality rates in our study are similar to the mean case fatality rates reported in countries belonging to the Organization for Economic Cooperation and Developmet (OECD) [39]. Like other studies [35], we found that the factors that contributed to explaining the likelihood of death at 30 days were pneumonia, age [36], speech disturbance, reduced level of consciousness and aphasia. Nevertheless, the two interventions selected did not have a significant effect on this outcome.

Our study has several limitations that could bias the results. We were unable to retrieve $17.5 \%$ of the sample because of the poor quality of medical registration in the patients' charts and the impossibility of retrieving the charts in one hospital after the 2010 earthquake. However, no difference was found by demographic characteristics and 30-day fatality between patients included in the sample and those not included. Therefore, we believe that the probability of selection bias is small and that our results reflect the reality of stroke care in these hospitals. Nevertheless, they may not reflect the care in hospitals in more isolated and less populated regions of the country, where standards could be lower and outcomes poorer, as shown in rural Australia by Cadilhac et al. [40].

Another limitation of this study is that we could not measure the effect of organized patient care (stroke units) as these facilities are only recently being implemented in some public hospitals in the Metropolitan Region of Santiago. In addition, we had no standardised summary measure of stroke severity such as the NIHSS and relied on other objective clinical measures at time of admission to account for stroke severity in our statistical models.

A strength of this study is that we were able to obtain data from hospitals admitting more that $50 \%$ of all patients with ischemic stroke in the country. We believe the findings of this study have good external validity for Chile as the age and sex distribution of the patients included was similar to those of the patients in the only community-based stroke study published to date the PISCIS study [3].

\section{Conclusions}

This is the first study to reveal the low implementation and adherence to evidence-based performance measures in hospitals admitting more than $50 \%$ of all ischemic stroke patients in Chile. These measures are part of the National Chilean Clinical Practice Guidelines for stroke, and reimbursement is guaranteed by health insurance agencies for some recommendations such as the use of CT scanning and thrombolytic therapy. We hope that these results will encourage decision makers to implement surveillance registries and modify the organization of health services that provide care to patients with stroke; in other countries, such actions have shown promising results in a relatively short term $[6,19,21]$. Furthermore, to increase the possibility of hyperacute interventions such as thrombolysis in a larger population, programs directed at lowering the barriers to prompt diagnosis and arrival at emergency departments should be designed and implemented.

\section{Competing interests}

The authors have no conflicts of interest.

\section{Authors' contributions}

LH made substantial contributions to the conception and design of the study, data collection and analysis, interpretation of the results and drafting of the manuscript. PL substantially contributed to the conception and design of the study, analysis and interpretation of the results and drafting of the manuscript. CV performed the data analysis, participated in data collection, interpretation and preparation of the results and contributed to the drafting of the manuscript. MC substantially contributed to the data analysis, preparation and interpretation of the results, and review of the manuscript. $\mathrm{RC}$ contributed to the study design, data collection and its monitoring and interpretation of the results and collaborated in the drafting of the manuscript. XC substantially contributed to the conception and design of the study and interpretation of the results and participated in the drafting of the manuscript. All the authors have reviewed and approved the final version of the manuscript.

\section{Authors' information}

We wish to highlight some relevant information about the authors: PL is a neurologist with a Master's in Public Health, and was the main researcher of the PISCIS project, a community-based study of the incidence of stroke in Chile, whose results were published in indexed journals. XC is and PhD with vast experience in research and health service systems. LH has a Master's in Public Health, is a Ph.D. candidate in Biomedicine and works at the School of Public Health in the Universidad Mayor of Chile.

\section{Acknowledgements}

This study was partially supported by the Ministry of Health of Chile which helped to find the study design and data collection, and by the University Mayor of Chile, which helped to fund the authors involved in data analysis and drafting of the manuscript, as well as its publication.

\section{Author details}

${ }^{1}$ School of Public Health, Facultad de Medicina, Universidad Mayor, Santiago, Chile. ${ }^{2}$ Neurology Service, Clínica Alemana de Santiago, Universidad del Desarrollo, Santiago, Chile. ${ }^{3}$ Department of Neurological Sciences, Facultad de Medicina, Universidad de Chile, Santiago, Chile. ${ }^{4}$ Department of Epidemiology and Evaluation, Hospital del Mar, Barcelona; IMIM (Hospital del Mar Medical Research Institute), Barcelona; Red de Investigación en Servicios de Salud en Enfermedades Crónicas (REDISSEC), Mar Teaching Hospital, 25-29 Passeig Marítim, 08003, Barcelona, Spain.

Received: 20 September 2012 Accepted: 27 February 2013 Published: 6 March 2013

\section{References}

1. Feigin VL, Lawes CM, Bennett DA, Barker-Collo SL, Parag V: Worldwide stroke incidence and early case fatality reported in 56 population-based studies: a systematic review. Lancet Neurol 2009, 8:355-369.

2. Johnston SC, Mendis S, Mathers CD: Global variation in stroke burden and mortality: estimates from monitoring, surveillance, and modelling. Lancet Neurol 2009, 8:345-354.

3. Lavados PM, Sacks C, Prina L, Escobar A, Tossi C, Araya F, et al: Incidence, 30-day case-fatality rate, and prognosis of stroke in Iquique, Chile: a 2- 
year community-based prospective study (PISCIS project). Lancet 2005, 365:2206-2215

4. Lavados PM, Sacks C, Prina L, Escobar A, Tossi C, Araya F, et al: Incidence, case-fatality rate, and prognosis of ischaemic stroke subtypes in a predominantly Hispanic-Mestizo population in Iquique, Chile (PISCIS project): a community-based incidence study. Lancet Neurol 2007, 6:140-148.

5. Govan L, Weir CJ, Langhorne P: Organized Inpatient (Stroke Unit) Care for Stroke. Stroke 2008, 39:2402-2403.

6. Cadilhac DA, Pearce DC, Levi CR, Donnan GA: Improvements in the quality of care and health outcomes with new stroke care units following implementation of a clinician-led, health system redesign programme in New South Wales, Australia. Qual Saf Health Care 2008, 17:329-333.

7. Donnan GA, Fisher M, Macleod M, Davis SM: Stroke. Lancet 2008, 371:1612-1623.

8. The National Institute of Neurological Disorders and Stroke rt-PA Stroke Study Group: Tissue plasminogen activator for acute ischemic stroke. N Engl J Med 1995, 333:1581-1587.

9. Adams HP Jr, del Zoppo G, Alberts MJ, Bhatt DL, Brass L, Furlan A, et al: Guidelines for the early management of adults with ischemic stroke: a guideline from the American Heart Association/American Stroke Association Stroke Council, Clinical Cardiology Council, Cardiovascular Radiology and Intervention Council, and the Atherosclerotic Peripheral Vascular Disease and Quality of Care Outcomes in Research Interdisciplinary Working Groups: the American Academy of Neurology affirms the value of this guideline as an educational tool for neurologists. Stroke 2007, 38:1655-1711.

10. Hacke W, Kaste M, Bluhmki E, Brozman M, Davalos A, Guidetti D, et al: Thrombolysis with alteplase 3 to 4.5 hours after acute ischemic stroke. N Engl J Med 2008, 359:1317-1329.

11. Lees KR, Bluhmki E, von Kummer R, Brott TG, Toni D, Grotta JC, et al: Time to treatment with intravenous alteplase and outcome in stroke: an updated pooled analysis of ECASS, ATLANTIS, NINDS, and EPITHET trials. Lancet 2010, 375:1695-1703.

12. Ministry of Health of Chile: Clinical Guidelines of acute ischemic stroke in adults (15 years and older). http://www.redsalud.gov.cl/archivos/guiasges/ isquemico.pdf.

13. The European Stroke Organization (ESO) Executive Committee and the ESO Writing Committee: Guidelines for Management of Ischaemic Stroke and Transient Ischaemic Attack 2008. http://www.eso-stroke.org/pdf/ ESO08_Guidelines_English.pdf.

14. National Stroke Foundation: Clinical Guidelines for Acute Stroke Management Australia: 2010. http://www.strokefoundation.com.au.

15. Eissa A, Krass I, Bajorek BV: Optimizing the management of acute ischaemic stroke: a review of the utilization of intravenous recombinant tissue plasminogen activator (tPA). J Clin Pharm Ther 2012, 37:620-629.

16. National Stroke Foundation: National Stroke Audit Acute Services Clinical Audit Report. 2011. http://www.strokefoundation.com.au.

17. Kleindorfer D, Xu Y, Moomaw CJ, Khatri P, Adeoye O, Hornung R: US geographic distribution of rt-PA utilization by hospital for acute ischemic stroke. Stroke 2009, 40:3580-3584.

18. Wasay M, Barohi H, Malik A, Yousuf A, Awan S, Kamal AK: Utilization and outcome of thrombolytic therapy for acute stroke in Pakistan. Neurol SCi 2010, 31:223-225.

19. Fonarow GC, Reeves MJ, Smith EE, Saver JL, Zhao X, Olson DW, et al: Characteristics, performance measures, and in-hospital outcomes of the first one million stroke and transient ischemic attack admissions in get with the guidelines-stroke. Circ Cardiovasc Qual Outcomes 2010, 3:291-302.

20. Schwamm LH, Fonarow GC, Reeves MJ, Pan W, Frankel MR, Smith EE, et al: Get With the Guidelines-Stroke is associated with sustained improvement in care for patients hospitalized with acute stroke or transient ischemic attack. Circulation 2009, 119:107-115.

21. Abilleira S, Ribera A, Sanchez E, Tresserras R, Gallofre M: The Second Stroke Audit of Catalonia shows improvements in many, but not all quality indicators. Int I Stroke 2012, 7:19-24.

22. Teasdale $G$, Jennett B: Assessment of coma and impaired consciousness. A practical scale. Lancet 1974, 2:81-84.

23. National Institute of Statistics: Mortality Trends in Chile of death causes and age, 1990-2007. http://www.ine.cl/canles/menu/publicaciones/ calendario_de_publicaciones/pdf/010211evo90_70_010211.pdf.
24. Saposnik G, Hill MD, O'Donnell M, Fang J, Hachinski V, Kapral MK: Variables associated with 7-day, 30-day, and 1-year fatality after ischemic stroke. Stroke 2008, 39:2318-2324.

25. Reid JM, Gubitz GJ, Dai D, Kydd D, Eskes G, Reidy Y, et al: Predicting functional outcome after stroke by modelling baseline clinical and $\mathrm{CT}$ variables. Age Ageing 2010, 39:360-366.

26. Landis JR, Koch GG: The measurement of observer agreement for categorical data. Biometrics 1977, 33:159-174.

27. George MG, Tong X, McGruder H, Yoon P, Rosamond W, Winquist A, et al: Paul Coverdell National Acute Stroke Registry Surveillance - four states, 2005-2007. MMWR Surveill Summ 2009, 58:1-23.

28. Saposnik G, Black SE, Hakim A, Fang J, Tu JV, Kapral MK: Age disparities in stroke quality of care and delivery of health services. Stroke 2009 40:3328-3335

29. Heuschmann PU, Kolominsky-Rabas PL, Misselwitz B, Hermanek P, Leffmann C, Janzen RW, et al: Predictors of in-hospital mortality and attributable risks of death after ischemic stroke: the German Stroke Registers Study Group. Arch Intern Med 2004, 164:1761-1768.

30. Sposato LA, Esnaola MM, Zamora R, Zurru MC, Fustinoni O, Saposnik G: Quality of ischemic stroke care in emerging countries: the Argentinian National Stroke Registry (ReNACer). Stroke 2008, 39:3036-3041.

31. Kim JS, Lee KB, Roh H, Ahn MY, Hwang HW: Gender differences in the functional recovery after acute stroke. J Clin Neurol 2010, 6:183-188.

32. Wang Y, Liao X, Zhao X, Wang DZ, Wang C, Nguyen-Huynh MN, et al: Using recombinant tissue plasminogen activator to treat acute ischemic stroke in China: analysis of the results from the Chinese National Stroke Registry (CNSR). Stroke 2011, 42:1658-1664.

33. Prasad K, Dash D, Kumar A: Validation of the Hindi version of National Institute of Health Stroke Scale. Neurol India 2012, 60:40-44.

34. Lakshminarayan K, Tsai AW, Tong X, Vazquez G, Peacock JM, George $M G$, et al: Utility of dysphagia screening results in predicting poststroke pneumonia. Stroke 2010, 41:2849-2854

35. Westendorp WF, Nederkoorn PJ, Vermeij JD, Dijkgraaf MG, van de Beek D: Post-stroke infection: a systematic review and meta-analysis. BMC Neurol 2011, 11:110.

36. Finlayson O, Kapral M, Hall R, Asllani E, Selchen D, Saposnik G: Risk factors, inpatient care, and outcomes of pneumonia after ischemic stroke. Neurology 2011, 77:1338-1345.

37. Aslanyan S, Weir CJ, Diener HC, Kaste M, Lees KR: Pneumonia and urinary tract infection after acute ischaemic stroke: a tertiary analysis of the GAIN International trial. Eur J Neurol 2004, 11:49-53.

38. Hubbard IJ, Harris D, Kilkenny MF, Faux SG, Pollack MR, Cadilhac DA: Adherence to clinical guidelines improves patient outcomes in Australian audit of stroke rehabilitation practice. Arch Phys Med Rehabil 2012, 93:965-971.

39. Organization for Economic Cooperation and Development: Health at a Glance 2001. OECD Indicators. http://www.oecd.org/health/healthataglance.

40. Cadilhac DA, Kilkenny MF, Longworth M, Pollack MR, Levi CR: Metropolitanrural divide for stroke outcomes: do stroke units make a difference? Intern Med J 2011, 41:321-326.

doi:10.1186/1471-2377-13-23

Cite this article as: Hoffmeister et al:: Performance measures for inhospital care of acute ischemic stroke in public hospitals in Chile. BMC Neurology 2013 13:23.

\section{Submit your next manuscript to BioMed Central and take full advantage of:}

- Convenient online submission

- Thorough peer review

- No space constraints or color figure charges

- Immediate publication on acceptance

- Inclusion in PubMed, CAS, Scopus and Google Scholar

- Research which is freely available for redistribution 